\title{
Dynamic pricing and resource allocation using revenue management for communications networks
}

\author{
Grigorios Zachariadis, Javier A Barria, Member, IEEE
}

\begin{abstract}
In this paper we develop a novel single-link multiple-classes-of-service framework where offered prices and QoS are allowed to be actively modified by the provider, depending on the demand and the congestion of the system. We obtain a solution to the problem using dynamic programming. The obtained solution is then extended to a network environment using a decomposition approach. The decomposition approach makes our solution scalable, since single-link solutions are used and minimal amount of information is explicitly exchanged. Assessments carried out for small networks show that the obtained income is improved between $2 \%-20 \%$ when compared to a static approach and to approaches where only price or only quality are allowed to be adaptive.
\end{abstract}

Keywords: Pricing, QoS, resource allocation, revenue management

\section{INTRODUCTION}

In packet networks congestion can degrade the perceived quality of service (QoS) therefore hinder the provider's conformance to QoS agreements. The exactly opposite situation (low utilisation) is also a problem: although it doesn't create problems to the functionality of the network, it causes problems to the provider since resources are not used optimally and therefore the investment in infrastructure does not bring the return it could.

In many published approaches, high congestion is dealt with by minimising its consequences. When congestion occurs the resources which are explicitly or implicitly dedicated to each user decrease, so that more users can be served (less rejected connection requests). In this way, users with high QoS requirements are effectively shut out of the system. Another technique to deal with congestion is increasing the prices of the resources when congestion occurs [15]. An alternative is letting the market free with no set prices, only rules as to how the prices are going to be determined through an auction[26][27][20][18], whereby a market reaches an equilibrium that reflects the supply and demand of services. In the approach here presented we consider that the provider is allowed to specify not only the price, but also the QoS for each offered service dynamically. This addition gives the provider more control over the network and brings better results in terms of its income and utilisation.

In this paper we assess different policies that a provider could follow in respect to the price and the quality (related to the resource allocation) of the offered services. We develop a novel algorithm, which yields the optimal combination of

The authors are with the Department of Electrical and Electronic Engineering,Imperial College London, London SW7 2BT, U.K. (e-mail: grigorios.zachariadis02@imperial.ac.uk; j.barria@imperial.ac.uk). prices and qualities of the services offered, depending on the current network state i.e. the number of active calls at the time of a new request for service.

This paper extends the approach we introduced in [32] by developing a solution for a network environment (as opposed to the single link case), by proposing alternative less computationally demanding approaches and by assessing all the proposed schemes. Furthermore, we note that in [31], the prices and quality were set according to the optimisation results considering the expected potential users mean arrival rate, but without adaptation for the current network state.

Moreover, [31] studies only static solutions (relation between demand, quality and prices), whereas in this paper an approach that considers the time of request for a new service and current network state (number od active calls and underlying demand) is introduced. This additional dimension of the problem is inspired by the airline industry pricing approach of revenue management [3], where the price for a product or service depends on the time it is purchased, its demand, its class and its availability. Hence, our approach extends the original revenue management principles to a telecommunications network environment. Note that when doing so, we can take advantage of the different nature of a telecommunications service in contrast to e.g. airfares, by allowing both price and quality (or, equivalently, resources per call) to be decision variables.

In the model here proposed, a provider has a specific amount of resources, and offers services belonging to different classes. Calls belonging to a class consume identical amount of resources at any specified time point. The amount of resources (and therefore the level of QoS) offered to each call of a class changes dynamically as a decision by the provider, and it cannot fall below a minimum level which is known to the users. Potential users are informed about the current QoS level and prices for all classes, to estimate the level of service they will receive. If one of the classes satisfies a user, he/she will request admission. If enough resources are available, then admission will be granted. The user immediately pays the advertised price, which is a one-off charge, and therefore any price changes after their admission do not affect them.

The rest of this paper is structured in the following manner. In Section II we briefly review existing publications relevant to our work. Section III describes the model used for our system. Section IV analyses the solution of the optimisation problem for a single link. Alternative solutions to the problem are developed and presented in Section V. In Section VI our solution is extended to a network. Section VII offers some preliminary numerical results from simulations, and the paper 
ends with a final remarks section.

\section{LITERATURE BACKGROUND}

Most published research uses pricing to control or regulate one of two things: either i) the rate at which new connection requests appear or ii) the data rate sent by existing connections or users. In this paper we use pricing to control the incoming rate of new connections.

When addressing the problems of resource allocation and pricing for telecommunication networks, we propose the application of revenue management principles from operations research. Revenue management [3] aims at maximizing income through the application of methods that predict customer behaviour and the optimisation of services availability and price. Revenue management has been successfully applied in industries like hotels or airlines, and it has been suggested that it can be applied in computer networks too [7]. We advocate that the service quality (QoS) is also a parameter which has to be considered in the case of telecommunication services.

A method considering pricing for multiservice networks is presented in [9]. The QoS of each service is given and guaranteed, and the problem of pricing is formulated as an expected income optimisation problem. In contrast, in this paper, we take into account QoS as a decision variable, which affects demand when determining the optimal prices.

Dynamic pricing policies for network services have also been widely investigated, and there is a great variety of propositions. Agent based [10], time-of-day [5], usage-based [30], threshold-based [21][6] and congestion dependent pricing [19][11] are some of them. The work of Paschalidis et al [15][16] on dynamic, congestion dependent pricing suggests that static (congestion-independent) pricing is asymptotically optimal, and therefore the benefits from dynamic pricing may not worth the extra complexity especially for large networks with many calls. A distributed mechanism for resource allocation, based on the pricing results of [15][16] is given in [17]. In contrast to the above approaches, the model used in this paper allows the provider to make dynamic decisions not only regarding the price, but also the quality of service of each class.

Regarding static pricing, in the "Paris Metro Pricing" (PMP) scheme [14] [22] two classes of services exist in the network, and each one has its own queue. Calls of the high class cost more than calls of the low class. In this way, fewer users request calls of the high class, and each call of the high class can thus use more resources, therefore receiving higher QoS. The PMP scheme exploits also the dependence of demand on prices. In this paper we extend the scheme proposed in PMP, as we also control and guarantee QoS.

Another study based on demand and pricing, is the work of McKie-Mason and Varian [12], which assumes that users pay a price depending on the congestion of the network and the utilisation, which in turn depend on the demand curve of individual users and the price. In contrast, in the approach presented in this paper we focus on pricing and QoS for classes of service, rather than individual users.

In the work presented in [2] and [1], the authors study the performance of the network under different bandwidth parti- tioning policies. It is observed via simulations that the prices and QoS offered have an effect to the income of a network provider. In [5] the combined problems of provisioning and pricing are studied. By provisioning the authors of [5] mean the procedure by which the provider purchases bandwidth, which then is divided between two classes. Time of day pricing is used, and making decisions in different timescales is evaluated. Provisioning is based in [5] on the actual cost of bandwidth which is assumed to be purchased by the provider. In our work we assume that the provider owns the resources or has purchased them in advance, and therefore provisioning is done under the constraint of limited bandwidth.

Resource negotiation and pricing (RNAP) [29] is a protocol to work with Diffserv. RNAP describes a resource negotiation framework which involves congestion-sensitive pricing and user rate adaptation. Prices consist of a holding charge (depending on time) and a usage charge (depending on volume). The authors in [29] use Langrangian multipliers to find the optimal solution. Our scheme differs from RNAP in that it does not require each user to react to price changes during a call, as the price for each user is constant and equal to the price at the beginning of the call. In our model the rate of each user needs not to be measured for billing purposes, but it has to be policed.

An important aspect of any pricing algorithm is the relationship between prices and demand (demand function). Little research is publicly available on this issue. An effort to experimentally explore the users' reaction to several pricing schemes is the INDEX experiment [4] [23]. The main conclusion of the experiment was that users changed their behaviour significantly under different pricing schemes.

In our approach we use effective bandwidths [8] to take into account the multiplexing gains of packet networks ${ }^{1}$. Compared to an approach where peak bandwidth is used, this has the advantage of taking into account statistical multiplexing gains, i.e. more calls can be accommodated to the network. The drawback of this is that since we take into account statistical multiplexing gains, the QoS guarantees offered are also statistical, which might not be desirable in some cases. Effective bandwidth plays a role in our model in two ways: the first one is to answer the question how many calls of each class can be accommodated given the specific amount of resources available. The second role is to answer the question what is the quality or satisfaction of the users for each different level of service. Hence, we have used effective bandwidth as a method to approximate the solution of these two problem. From the network point of view effective bandwidth is used as a method to evaluate whether a new call can be accepted and secondly to differentiate the QoS of each class by, e.g., being used in setting the weights of a a weighted fair queueing (WFQ) system. However, we note that in the approach presented in this paper, both of these functions could be performed using any other suitable method(s), depending on the scale of the system, the relevant information available and the processing power of a provider.

\footnotetext{
${ }^{1}$ The mapping of QoS requirements into effective bandwidth is beyond the scope of this paper.
} 


\section{SINGLE-LINK SERVICE AND DEMAND MODEL FOR DYNAMICALLY CHANGING QUALITY AND PRICES}

In this paper we use a framework similar to the one used in [31], with a modification in the fact that the provider can dynamically change prices and qualities. Consider a provider who offers calls of several classes. Each class represents a different service category in resource allocation. We assume that each call of the $i$ 'th class incurs a one-off charge of $p_{i}$. We use a value $q_{i}$ as a QoS index for the $i$ 'th class. The way $q_{i}$ is determined depends on the exact characteristics of the system we are studying. The index $q_{i}$ is equal to the amount of bandwidth $r_{i}$ allocated to each flow of the $i$ 'th $\operatorname{CoS}$, to show that the QoS of a flow is higher when more bandwidth is allocated to it. The amount of bandwidth assigned to flows of a class dictates the proportion of the total resources that flows of this class receive.

Our algorithms would assist a service provider in deciding how he/she will distribute the available bandwidth of its system among the users of the different classes and how many more users he/she can accept. Our proposal can rely on a WFQ algorithm (see e.g. [25]), where the weights for packets of each class depend on the number of active flows for the class and the allocated bandwidth per flow. Potential users of the network with specific requirements arrive according to a Poisson process and if the QoS and the price for a class are acceptable for them then they request admission for this specific class. In order to model the satisfaction of a potential user from prices and QoS, we have used a utility function which includes the price element, i.e. a highly priced product has a lower utility than a low priced product of the same usefulness. Hence we characterise a user's behaviour by the following utility function [24]:

$$
U_{i}(p, q, \gamma)=\left[\gamma\left(\hat{p}-p_{i}\right)+(1-\gamma) q_{i}\right] \theta\left(\hat{p}-p_{i}\right) \theta\left(q_{i}-\hat{q}\right)
$$

where $U_{i}(p, q, \gamma)$ is the user utility with respect to the $i$ 'th class, and $p_{i}$ and $q_{i}$ are the price and QoS for the $i$ 'th class, respectively. In this equation, $\hat{p}$ and $\hat{q}$ are the maximum price a user is willing to pay and the minimum QoS the user can tolerate, respectively. We assume that $\hat{q}=\eta \hat{p}$ ( $\eta$ constant) to reflect the fact that users who demand better QoS are willing to pay more. We assume that $\hat{p} \in[0,1], \hat{q} \in[0, \eta]$. Without loss of generality we use $\eta=1$ quality unit per price unit. We denote $\theta(x)$ a step function which is 0 for $x<0$ and 1 for $x \geq 0$. It is assumed that $\gamma$ is a weight that quantifies a user's relative sensitivity to service price and QoS. Combined with a minimum acceptable QoS for each user, our utility function (Fig. 1) can approximate a linearly increasing, a sigmoid or a step function, capturing three major classes of utility functions. The requirements of users $\hat{p}, \hat{q}$ and the distribution of the users with respect to those requirements play a very important role in our work, since it is through this distribution that the variables of price and QoS for a class affect the actual demand. For our simulations, this distribution is assumed to be uniform, unless stated otherwise.

Note that in (1), the second term $(1-\gamma) q_{i}$ is used instead of the normalized $(1-\gamma)\left(q_{i}-\hat{q}\right)$ in [24]. This change does not distort the underlying behaviour of the user since, on the

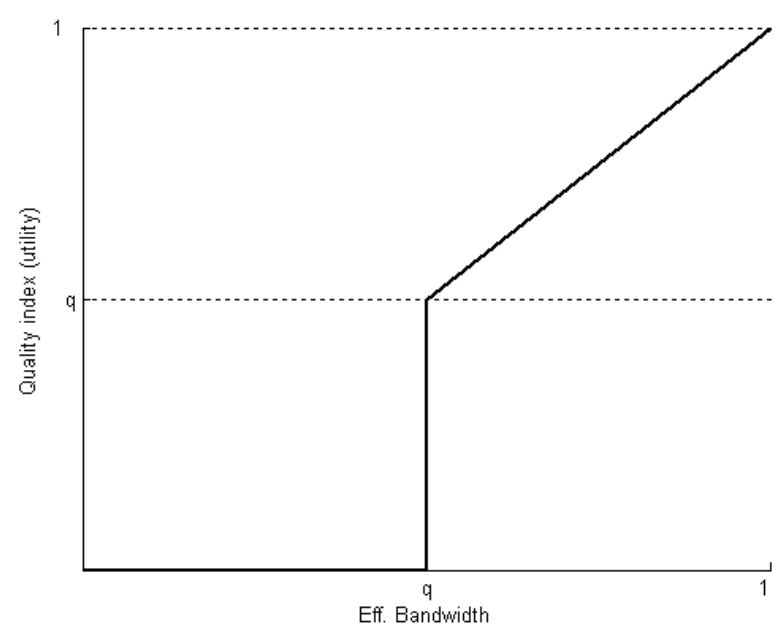

Fig. 1. How bandwidth affects quality for a user with minimum requirement $\hat{q}$.

one hand, whether a user will get utility greater than zero is only affected by the $\theta$ functions and, on the other hand, the issue of which class offers the user the greater utility is also not affected by the modified version (since for each user the utility is increased equally for all the classes by $(1-\gamma) \hat{q})$. The reason behind the change is that with the original model suggested in [24], a user would have near zero utility for a call which just provided his/her minimum requirement, and, the model would not be able to be used with a target like e.g. welfare (instead of income) maximisation (where the total utility would be used as the target to be maximised) making our approach more extendable.

In the model here presented, the user pays a fee at the beginning of his/her call and this is his/her only obligation that we take into account. Because of this, future fluctuations in price do not affect him/her. We consider that potential users use the current QoS as an estimation of the QoS they are going to receive during their connection. However, although the QoS is guaranteed above a minimum level, there is no guarantee that the current QoS will be the actual QoS the users will experience during the whole duration of their connection.

We assume that the call durations are exponentially distributed with departure rate $\mu_{i}$ for the $i$ 'th class. Forecasts of $\mu_{i}$ are considered known to the provider by previous observations. For simplicity we have assumed that $\mu_{i}=1$ for all $i$.

A user chooses to request connection (i.e. initiation of a flow of packets) for a specific class if his/her utility is positive $\left(U_{i}>0\right)$ for this class. In the event that $U_{i}$ is positive for more than one classes the user chooses the one with the highest utility.

Using the distribution of the potential users with respect to their quality demands and maximum tolerated price we can calculate the arrival rate of potential customers $\lambda_{k}$ for each class $k$ for each value of $p_{k}$ and $q_{k}$. The arrival rate of users with requirements $x$ such that $q_{k}>x>p_{k}$ is also the arrival rate of users requesting connection in class $k$, when they can only be satisfied by this class. If the probability density function of the distribution of users with respect to 


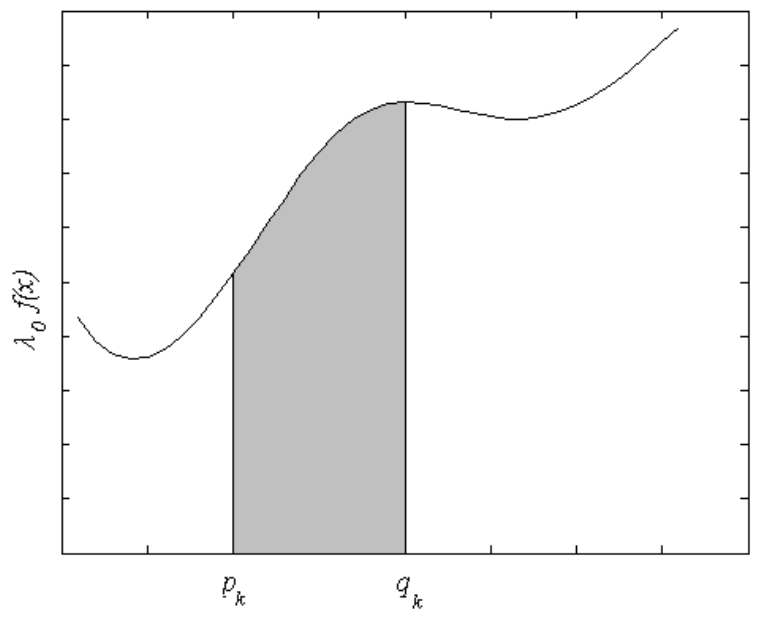

Fig. 2. Arrival rate of users with requirements between $p_{k}$ and $q_{k}$.

their requirements is $f(x)$, and the mean total arrival rate of users is $\lambda_{0}$, then the arrival rate of users with requirements $x$ such that $q_{k}>x>p_{k}$, i.e. the arrival rate for class $k$ users, if there are no other classes satisfying any of the users with such requirements, is given by the following equation (shaded area in Fig. 2):

$$
\lambda_{k}=\lambda_{0} \int_{p_{k}}^{q_{k}} f(x) d x
$$

For example, in the case of uniform distribution, the above equation is reduced to $\lambda_{k}=\lambda_{0}\left(q_{k}-p_{k}\right)$. If the prices and qualities of two classes are such that some users might be satisfied by both classes, we will say that they overlap. In this case one part of the users which are satisfied by both classes will select one class and another part will select the other. For a service provider, rejecting a call is undesirable, since it means that a customer is unsatisfied, and may switch to another provider. To avoid high numbers of rejected calls we can assume that there is a penalty for each rejected call. This penalty symbolises the expected damage caused by each rejected call to the provider.

Note that the assumption of having the same $\mu_{i}$ for all classes can be relaxed. If we assume that the average call duration is class dependent, we should also take into account the call duration when modelling the preferences of a user. Specifically, the price requirement should be substituted by a price per time unit requirement. Furthermore, the one-off charge should also be substituted by a time-based charging. Note, however, that even in this case, the average (expected) payment of a call of a class corresponds to the price of a call of average duration for the class it belongs to, and therefore, the algorithms we present do not need to be changed. Finally, since the quality of a call is only allowed to fluctuate within specific limits, we can assume that such fluctuations do not greatly affect the connection duration. This is especially true for the case of applications of variable bit rate, such as teleconferences, and streaming audio or video, where the effect of small quality fluctuations to the duration can be considered minimal.

\section{OPTIMAL DYNAMIC POLICY FOR PRICING AND QUALITY}

The operation of a network provider is a complex affair where several targets have to be met simultaneously. In our work we concentrate on the target of income maximisation, which, due to the fact that the marginal costs of a network provider are minimal, can be translated to profit maximisation [7]. Any telecommunications business will need to assess their income as part of their operations viability and hence we investigate this aspect here. Other targets, which have been suggested in the research community are: i) Welfare maximisation: $\max \sum u_{i}$ (where $u_{i}$ is the utility of user $i$ ), and ii) Fairness: $\max \left(\min _{i} u_{i}\right)$ [25]. We note that the target of welfare maximisation could be achieved within the framework presented in this work, where instead of price we would consider the utility of the user. Towards this direction, an extension to the application of revenue management principles has been presented in [13], where the target is maximising the utility while staying within specific revenue constraints.

Our target is to create an algorithm to set the prices and the QoS of services so that the long-term expected income rate of the provider is maximised. If penalties for rejection are taken into account, the expected income rate is

$$
\lim _{T \rightarrow \infty} \frac{1}{T} E\left[\int_{0}^{T}\left[\sum_{i \notin C} \lambda_{i}(t) p_{i}(t)-\sum_{i \in C} c \lambda_{i}(t) p_{i}(t)\right] d t\right]
$$

where $C(t)$ is the set of classes for which calls are rejected and $c$ is a penalty factor.

The constraints in our problem are:

- $\sum n_{i} r_{i}<W$

- $r_{i} \geq r_{i, \min }$

where $r_{i, \min }$ is the minimum bandwidth allocated to each call of class $i, n_{i}(t)$ is the number of ongoing calls for class $i$ at time $t$ and $W$ is the total capacity of our link. The first constraint implies that we employ a complete sharing scheme among the classes. That is to say, unoccupied bandwidth can be taken up by calls of any class. If we have available bandwidth for a requested call, we accept it, rather than reject it in the hope that a more profitable call will appear. If such a rejection would be more profitable, our algorithm would set the price of the non-profitable call so high that there would be no demand for it.

The state of our system is denoted by the vector $\mathbf{n}(t)=$ $\left(n_{1}, \ldots, n_{k}, \ldots, n_{K}\right)$, where $K$ is the total numbers of classes. An arrival of class $k$ will lead to the state $\left(n_{1}, \ldots, n_{k}+\right.$ $\left.1, \ldots, n_{K}\right)$, whereas a departure leads us to $\left(n_{1}, \ldots, n_{k}-\right.$ $\left.1, \ldots, n_{K}\right)$. The state of the system changes when the number of calls of any class changes, due to an arrival or a departure. We have assumed a Poisson arrival process and exponentially distributed service time. With these assumptions the state transitions depend only on the current state, and therefore can be modelled as a Markov process.

The reward rate and the time until next transition at each state $\mathbf{n}$ are bounded functions of $\mathbf{p}, \mathbf{r}$, where $\mathbf{p}(t)=$ 
$\left(p_{1}, \ldots, p_{K}\right)$ and $\mathbf{r}(t)=\left(r_{1}, \ldots, r_{K}\right)$. The same is true for the total transition rate out of any state.

The process $n(t)$ is a continuous-time Markov chain. Since the total transition rate out of any state is bounded by

$$
\nu=\sum_{i}\left(\lambda_{i}+\mu_{i} \frac{W}{r_{i \min }}\right)
$$

this Markov chain can be uniformised. If we denote by $\pi_{\mathbf{n}, \mathbf{n}^{\prime}}$ the probability of transition from state $n$ to state $n^{\prime}$ in the uniformised chain, the set of Bellman equations (one for each state $\mathbf{n})$ is:

$$
J^{*}+h(\mathbf{n})=\max _{\mathbf{p}, \mathbf{r}}\left[E R_{n}+\sum \pi_{\mathbf{n}, \mathbf{n}^{\prime}} h\left(\mathbf{n}^{\prime}\right)\right]
$$

where $J^{*}$ is the optimal average reward, $h(\mathbf{n})$ is the relative reward for being in state $\mathbf{n}$ and $E R_{n}$ is the expected rate of income in state $n$ (for simplicity, the penalty factors are omitted):

$$
E R_{n}=\sum_{i \notin C(\mathbf{n})} \lambda_{i}(\mathbf{p}, \mathbf{r}) p_{i}
$$

$C(\mathbf{n})$ is the set of classes for which an admission request will be refused at state $\mathbf{n}$ and $\lambda_{i}$ is the mean arrival rate of requests for calls of class $i$. Substitution leads to the following set of Bellman equations (one for each state $\mathbf{n}$ ):

$$
\begin{aligned}
J^{*}+h(\mathbf{n})= & \left.\left.\begin{array}{l}
\sum_{i \notin C(\mathbf{n})} \lambda_{i}(\mathbf{p}, \mathbf{r}) p_{i}+\sum_{i \notin C(\mathbf{n})} \frac{\lambda_{i}(\mathbf{p}, \mathbf{r})}{\nu} h\left(\mathbf{n}+\mathbf{e}_{\mathbf{i}}\right) \\
\max _{\mathbf{p}, \mathbf{r}}^{K}
\end{array}\right] \begin{array}{l}
\sum_{i=1}^{K} \frac{n_{i} \mu_{i}}{\nu} h\left(\mathbf{n}-\mathbf{e}_{\mathbf{i}}\right)+\sum_{i=1}^{K} \frac{n_{i} \mu_{i}}{\nu} h\left(\mathbf{n}-\mathbf{e}_{\mathbf{i}}\right) \\
+\left(1-\sum_{i \notin C(\mathbf{n})} \frac{\lambda_{i}(\mathbf{p}, \mathbf{r})}{\nu}-\sum_{i \notin C(\mathbf{n})} \frac{n_{i} \mu_{i}}{\nu}\right) h(\mathbf{n})
\end{array}\right](7)
\end{aligned}
$$

In the above equation, $\mathbf{e}_{\mathbf{i}}$ is the vector with all other elements equal to 0 and element $i$ equal to 1 .

Once Bellman's equation is solved, an optimal policy is obtained by choosing at each state $\mathbf{n}$ the price and bandwidth per call vectors $\mathbf{p}, \mathbf{r}$ that maximise the right-hand side of (7). The solution to Bellman's equation and a resulting optimal policy can be computed using classical dynamic programming algorithms. However, the computational complexity increases with the size of the state space, which is exponential in the number of classes $K$.

Note here that this approach requires that the provider has knowledge of the level of demand for each different set of prices and qualities. This can rely on the assumption that historical data is available or that underlying assumptions are the outcome of the historical observations (because the policy proposed here is dynamic, several different values for prices and qualities are used, and hence historic information will eventually come available). There are practicable steps which can make our approach also applicable to an uncertain environment. The approach we would consider first is a reinforcement learning approach (e.g., [28, 33, 34]), where the decision system learns automatically the optimal actions corresponding to each state. Such approaches do not need to be based on historical data, as their are based on observation of current data, associating different network states and decisions to results by observation. Bertsikas offers theoretical guarantees for their convergence to the optimal solution. There is a wide variety of methods which can be used, and their assessment is an interesting exercise in applied maths, which however is outside of the focus of this paper.

Note that we use the term policy to refer to the prices and qualities set by the provider for each state of the network. We will study and evaluate 4 policies I. Policy 1 refers to the optimal policy under the condition that the prices and qualities for each class are the same regardless of the state of the network [31]. Policy 1 can also be considered to represent an alternative, sub-optimal policy, where the values of prices and qualities are fine-tuned periodically according to measurements of demand and estimation of the derivatives of demand to price and quality. Policy 1 is the optimal policy and, hence its best possible upper bound. Policy 2 is the optimal policy under the condition that quality for each class is the same for each state of the network [15]. Policy 3 is the optimal policy when the price for each class is the same for each state of the network (which we develop in the next section for comparison purposes). Policy 4 is the optimal policy when both prices and qualities are allowed to be dynamic, i.e. depending on the state of the network.

\section{Alternative SOLUtions to THE PROBLEM}

Although the solution of (7) requires great computational time, alternative solutions, less demanding computationally, can be calculated using simplified methods, yielding results which are below optimal. In this section we present these solutions.

\section{A. Dynamic quality/price only}

An alternative to allowing the provider to dynamically control the price and the quality of the offered services, is allowing the provider to control dynamically only the price, while the quality remains static, or only the quality, while the price remain static (see Table I). The dynamic price/static quality policy (Policy 2) has already been studied in the literature [15]. We develop here the dynamic quality/static price policy (Policy 3) for the purpose of comparison. The optimal decision can be calculated in a way similar to the one we analysed in Section IV. So for Policy 3 the Bellman equation has the form:

$$
\begin{aligned}
J^{*}+h(\mathbf{n})= & {\left[\begin{array}{l}
\sum_{i \notin C(\mathbf{n})} \lambda_{i}(\mathbf{p}, \mathbf{r}) p_{i}+\sum_{i \notin C(\mathbf{n})} \frac{\lambda_{i}(\mathbf{p}, \mathbf{r})}{\nu} h\left(\mathbf{n}+\mathbf{e}_{\mathbf{i}}\right) \\
\max _{\mathbf{r}}^{K}\left[\sum_{i=1}^{K} \frac{n_{i} \mu_{i}}{\nu} h\left(\mathbf{n}-\mathbf{e}_{\mathbf{i}}\right)+\sum_{i=1}^{K} \frac{n_{i} \mu_{i}}{\nu} h\left(\mathbf{n}-\mathbf{e}_{\mathbf{i}}\right)\right. \\
+\left(1-\sum_{i \notin C(\mathbf{n})} \frac{\lambda_{i}(\mathbf{p}, \mathbf{r})}{\nu}-\sum_{i \notin C(\mathbf{n})} \frac{n_{i} \mu_{i}}{\nu}\right) h(\mathbf{n})
\end{array}\right] \text { (8) } }
\end{aligned}
$$

Compared to (7),the distinguishing factor here is that the maximisation is over the resources per call of each class $r$, while the prices $p_{i}$ are constant for each class of service, i.e. it is not a decision variable in the Bellman equation. 


\begin{tabular}{|c|c|c|c|}
\hline & Prices & QoS & Reference \\
\hline Policy 1 & Static & Static & {$[31]$} \\
Policy 2 & Dynamic & Static & Section V, [15] \\
Policy 3 & Static & Dynamic & Section V \\
Policy 4 & Dynamic & Dynamic & Section IV \\
\hline
\end{tabular}

TABLE I

THE FOUR POLICIES REGARDING STATIC OR DYNAMIC SETTING OF PRICING AND QUALITY

\section{B. Myopic solutions}

A set of alternative solutions involves applying a greedy policy which only maximises immediate rewards, without estimating rewards in the future. This is the policy which emerges if we disregard the value function in (7) or, equivalently, if we consider that there is a discount factor equal to 0 for future income, which means that future income is not taken into account. The myopic solutions do not take into account the opportunity cost of accepting a connection, i.e. the fact that accepting a connection now may result in having to reject a, potentially more profitable, connection at a later time. The equation which describes the maximisation relevant to this policy is the following:

$$
J^{*}=\max _{\mathbf{p}, \mathbf{r}} \sum_{i \notin C(\mathbf{n})} \lambda_{i}(\mathbf{p}, \mathbf{r}) p_{i}
$$

Note that a myopic version exists for the solution we proposed in Section IV, where prices and quality are dynamic, as well as for the solutions of Section V-A, where one set of the decision variables is static and the other dynamic. In the case where the prices are set myopically, the prices are set relatively low, thus increasing the expected arrival rate of connection requests. In the case where quality is set myopically, quality for each class will usually be the highest possible quality which is such that an arriving connection request can be accepted. In this way, the incoming rate of new connections is increased, and unnecessary rejections do not take place.

\section{Computational Complexity and Practical considerations}

If we denote $K$ the number of classes, $Q$ the quantisation step and $S$ the maximum number of calls of each class which can be accommodated, then the computational complexity of the policies is (for the single link case) as shown in Table II. It is well known that Dynamic programming is not suitable for online implementation due its complexity (see e.g. [35]), so the policies can be calculated off-line, and then retrieve the solution and applied them in real-time. An alternative, on-line approach could be a reinforcement learning scheme, where the function $J^{*}$ is approximated by a polynomial, and the values that have to be updated are not the $J^{*}$ corresponding to each state (whose number is $O\left(K^{M}\right)$, with $M$ being approximately $\left(\Lambda^{K}\right)$, where $\Lambda$ is the maximum number of calls of the highest class which can be accommodated) but the factors of the polynomial, which are $\mathrm{O}\left(K^{R}\right)$, where $R$ is the degree of the polynomial used.

For the system described in this paper, the timescale for the acceptance of a new call is approximately the time until another call requests admission or departs the system. If the network changes states more quickly, rendering this approach unstable, an alternative would be to aggregate the network states in groups and using thresholds for entering and leaving network states, so that the prices, qualities and admission decisions do not change in the time that is needed until a call is initiated. Furthermore, such an alternative approach would also yield benefits in terms of computational requirements, but would result in a reduced benefit compared to a static approach. Another, simpler, approach is to reserve the resources before the user confirms that he initiates a new call, i.e. from the time a user requests information regarding pricing and quality until he confirms or rejects the initiation or a reasonable amount of time passes.

\section{EXTENSION TO NETWORKS}

All the policies described so far have been developed for single links only. Here we develop a model that includes the cases in which flows can use multiple links. Our network model is similar to the one used in [31]. We consider a network with a set $L$ of links and a set $T$ of possible call routes. Potential calls for route $R \in T$ arrive according to a Poisson process with mean rate $\lambda_{0, R}$. Each potential user has specific quality and price requirements. If a specific class of service on route $R$ is satisfying the potential user, the user will request admission. If enough resources are available on all links belonging to $R$, the request will be accepted, otherwise it will be blocked.

\section{A. Centralised approach}

The centralised way of solving this problem, according to the method developed in Section IV is to consider each set of calls which traverse the same route and belong to the same class, as an independent set of calls which receive the same treatment (in terms of price and quality). Effectively, the classes of service in (7) should be substituted by sets of connections which receive to the same QoS and also traverse the same route. Then the Bellman equation for this problem be very similar to (7):

$$
\begin{aligned}
J^{*}+h(\mathbf{n})= & \left.\begin{array}{l}
\sum_{i \notin C(\mathbf{n})} \lambda_{i}(\mathbf{p}, \mathbf{r}) p_{i}+\sum_{i \notin C(\mathbf{n})} \frac{\lambda_{i}(\mathbf{p}, \mathbf{r})}{\nu} h\left(\mathbf{n}+\mathbf{e}_{\mathbf{i}}\right) \\
\max _{\mathbf{r}, \mathbf{p}}^{K|T|} \\
+\sum_{i=1}^{K|T|} \frac{n_{i} \mu_{i}}{\nu} h\left(\mathbf{n}-\mathbf{e}_{\mathbf{i}}\right)+\sum_{i=1}^{K|T|} \frac{n_{i} \mu_{i}}{\nu} h\left(\mathbf{n}-\mathbf{e}_{\mathbf{i}}\right) \\
+\left(1-\sum_{i \notin C(\mathbf{n})} \frac{\lambda_{i}(\mathbf{p}, \mathbf{r})}{\nu}-\sum_{i \notin C(\mathbf{n})} \frac{n_{i} \mu_{i}}{\nu}\right) h(\mathbf{n})
\end{array}\right]
\end{aligned}
$$




\begin{tabular}{|c|c|}
\hline Policy 1 & $O(1)$ \\
Policy 2 & $O\left(S^{K}(1 / Q)^{K}\right)$ \\
Policy 3 & $O\left(S^{K}(1 / Q)^{K}\right)$ \\
Policy 4 & $O\left(S^{K}(1 / Q)^{2 K}\right)$ \\
\hline
\end{tabular}

TABLE II

COMPUTATIONAL COMPLEXITY OF THE FOUR POLICIES

The difference to (7) is that each element of $\mathbf{n}$ now represents the number of connections on a specific route and QoS classification, and, similarly, that the values of $\mathbf{q}, \mathbf{p}, \mathbf{e}_{\mathbf{i}}$ refer to such sets of connections. $K$ is now the number of different QoS classifications allowed for each route, and $|T|$ the number of routes. The state space is now $O\left(e^{K|T|}\right)$, which is a dramatic increase compared to the case of a single link, for which the state space is $O\left(e^{K}\right)$.

There are several disadvantages of this approach which make it impractical. Firstly, the scale of the problem is immense even for very small networks. Secondly, even if a solution was obtainable, its implementation would be hindered by the fact that calls which traverse a link while they belong to a different route, but to the same class of service will probably have different quality requirements. For this reason, the different levels of quality that must be offered on each link will be much more than the number of classes, thus canceling a lot of the simplicity and the scalability that classes offer. Furthermore, this approach to the problem assumes that all links belong to a single administrative domain and thus act co-operatively, and thus would not be applicable to a multi-domain environment. Given the disadvantages of the centralised approach, we have developed and proposed a decomposition approach which tackles the above problems.

\section{B. Decomposition approach}

The aim of this approach is to decompose the centralised problem to many smaller, local problems, whose scale is such that they are solvable. This is achieved by solving the problem of pricing and setting the quality independently for each link, and then combining the prices and the qualities of the links along each route to calculate the service characteristics for an end-to-end connection. Note that each link adopts the solution which is optimal for it self based on the information it has, and therefore this approach can also be applied in the case of networks with more than one administrative domain. Each call belongs to a specific class, which is the same for all the links it crosses. This means that a call cannot belong to class $i$ of a link and class $j, j \neq i$ of another link.

Since in this work we have assumed that bandwidth is the QoS metric of interest, the overall quality of a route is the worst of the qualities of the links traversed by a route:

$$
q_{R, i}=\min _{l \in R} q_{l, i},
$$

where $q_{R, i}$ is the quality of route $R$ for QoS level $i$, and $q_{l, i}$ is the quality of link $l$ for QoS level $i$. Since each user requests connection for a specific route, the effect of selecting different routes is not studied in this paper. Users requesting connections of longer distance are aware of that and they have factored in the implications of that to their requirements. For example, a delay sensitive user, might achieve his/her desired QoS by having a quality requirement $q_{S}$ over a short route, or a quality requirement $q_{L}>q_{S}$ when the route of interest is longer.

For the problem of relating the price of a route to the price of each link independently, in our approach we consider that users are prepared to pay for the resources they are using, and therefore, the price of a route is the sum of the prices of each link that it traverses.

$$
p_{R, i}=\sum_{l \in R} p_{l, i}
$$

For the solution of the problem on each link, a local estimation of the incoming rate of connections is used, which is based on the approximation that all the other links have, at any time, the same values of prices and QoS as the one for which the calculations are made. This means that the value of the incoming rate of connections $\lambda_{R, i}$ of route $R$ and QoS classification $i$ is based on the approximations $q_{R, i}=q_{l, i}$ and $p_{R, i}=|R| p_{l, i}$, where the calculations are made for the local problem of link $i$ and $|R|$ the number of links in route $R$. This is a good approximation especially in the case when the links are relatively large, because in smaller link each arrival or departure makes a big difference in utilisation, leading to great fluctuations of prices and qualities, thus rendering the above approximations less accurate. Simulations results presented in Section VII indicate that the dynamic pricing and quality approach improves the income considerably in all cases studied. Using the approximate values of incoming connection request arrival rate for each route, an overall request arrival rate estimate for the link is used:

$$
\lambda_{l k}^{(l)}=\sum_{R: l \in R} \lambda_{0, R}\left(q_{l k}-p_{l k}\right)=\lambda_{0 l}\left(q_{l k}-p_{l k}\right)
$$

where $\lambda_{l k}^{(l)}$ is the local estimate (at link $l$ ) for the connection request arrival rate and

$$
\lambda_{0 l}=\sum_{R: l \in R} \lambda_{0, R}
$$

In (13) a uniform distribution of users with respect to their requirement has been used, leading, through (2), to the following equations:

$$
\lambda_{R k}=\lambda_{0, R}\left(q_{l k}-p_{l k}\right)
$$

For other distributions, (13) is replaced by the more general equation: 


$$
\lambda_{l k}^{(l)}=\lambda_{0 l} \int_{p_{l k}}^{q_{l k}} f(x) d x
$$

In any case, the problem to be solved is that of a single link, and we can use the solutions presented in the previous sections.

\section{Computational complexity}

For the case of multiple links, denote $|L|$ the number of links and $|T|$ the number of routes. For a centralised approach Policies 2 and 3 are $O\left(S^{K|T|}(1 / Q)^{K|T|}\right)$ and Policy $4 O\left(S^{K|T|}(1 / Q)^{2 K|T|}\right)$, since the number of classes to be taken into account is now $K|T|$, considering that classes are now differentiated not only by their quality but also by the route. For a distributed approach, the single link problem has to be solved on each link, and therefore, Policies 2 and 3 are $O\left(|L| S^{K}(1 / Q)^{K}\right)$ and Policy 4 is $O\left(|L| S^{K}(1 / Q)^{2 K}\right)$. This high complexity is the so called "curse of dimensionality" of dynamic program, and known methods to ameliorate its effect is the use of approximate methods, i.e. approximating the reward function with a simple function (i.e. linear or quadratic) and estimating its parameters through reinforcement learning, or reducing the number of states by grouping states together.

The fact that the solution we propose is distributed, means that the computational load required for the calculation of the optimal qualities and prices can be done in a distributed manner as well (only the local estimation of service request $\lambda_{0}$ at the link level is needed). To further reduce the computational burden, a hybrid approach could be adopted: for links of high scale (large capacity accommodating many users), and links of low utilisation, a static policy could be used (Policy 1) which has minimal computational requirements, and yields results close to dynamic policies for such cases. This approach could also include other links which do not contribute significantly to the total income of the provider, and therefore the increase in income does not justify the extra computational resources needed. For the rest of the links, a dynamic policy could be used. Thus an optimal balance can be achieved which is even more scalable and also benefits from the improvements of our proposal.

\section{NUMERICAL RESULTS}

For our preliminary tests we consider the case of a single link with bandwidth $W$. Potential users arrive to the network with rate $\lambda_{0}$ and check the available services. Each arriving user is characterised by a random (uniformly distributed) value which is the maximum price and minimum QoS he/she wants. The distribution we select for the users' requirements does not play a significant role in the efficiency of our proposed approach, because the algorithm used to calculate the optimal prices and qualities is based on extensive search (brute force) calculation of demand for each different set of prices and qualities, and therefore always yields a nearoptimal solution tailored to the distribution used. This includes integrable functions. This includes functions with Dirac's delta elements essentially making this applicable in cases where there is great concentration or change of demand at specific values of price and quality, due to the existence of a widespread application with specific requirements or due to the existence of competitors. In such cases, a small change in quality or price may induce a big change in demand at certain levels. Note that using, e.g., a reinforcement learning technique, the optimisation could be based on observed, rather than calculated demand.

$\mathrm{He} / \mathrm{she}$ will request admission for a call of the class with the maximum utility. The provider will accept the call, if there is enough bandwidth. Whenever the state of the system changes, the provider sets the price (for new calls) and the QoS for each class in the optimal value for the new state, which has been calculated offline through dynamic programming. Prices and resources can be set for each class either statically or dynamically, using one of the four policies shown on Table I.

The minimum bandwidth offered for calls of each class is shown on Table III. The maximum allowed price is 1 . For Policy 1, which includes static assignment of prices and bandwidth, the optimal values can be calculated analytically or using approximations (see [31]). For the policies where only prices or only QoS have to be set statically, there is no analytical solution and we used the values which are optimal for the all-static case. Simulations show that such assignment is near optimal. We quantise the possible values of price and QoS with a step of 0.05 for the case where all decisions are dynamic and 0.01 for the case where only price or only QoS is dynamic.

In each set of results we simulate the operation of a network, under a steady $\lambda_{0}$ (i.e. not changing with time) for a specific long duration (100,000 seconds). The optimal values for prices and QoS corresponding to each state are calculated beforehand. Dynamic programming problems (for policies 2,3 and 4) have been solved using the method of value iteration [28]. Note that the following formula has been used for the calculation of income from simulations in this paper:

$$
\sum_{i=1}^{N} \bar{p}_{i} / T_{s}
$$

where $\bar{p}_{i}$ here denotes the price paid by user $i, T_{s}$ the total time simulated and $N$ the total number of accepted users during $T_{s}$.

\section{A. Results}

In Figure 3 we observe that Policy 4 outperforms all three other policies by quite a large margin. The tradeoff for this is the fact that the complexity of Policy 4 is much larger. As the incoming demand increases, the improvement in results from using Policy 4 increases. This is expected because when the demand is very low the congestion doesn't play any role (it doesn't exist) and so there is no need or purpose in differentiating the $p$ and $q$ depending on the current state.

With policies 2, 3 and 4 the number of rejections is not a useful measure, because in some of the states where a call would be rejected, the prices and qualities may be set in a way that no user will request admission for a class that would be rejected. In order to have a fair comparison, we therefore do not use the penalties for rejected calls for the calculation of income. We suggest to consider the number of 


\begin{tabular}{|c|c|}
\hline Class & Min. Bandwidth \\
\hline Class 1 & 0.75 \\
Class 2 & 0.50 \\
Class 3 & 0.25 \\
\hline
\end{tabular}

TABLE III

MINIMUM BANDWIDTH FOR CALLS OF EACH CLASS

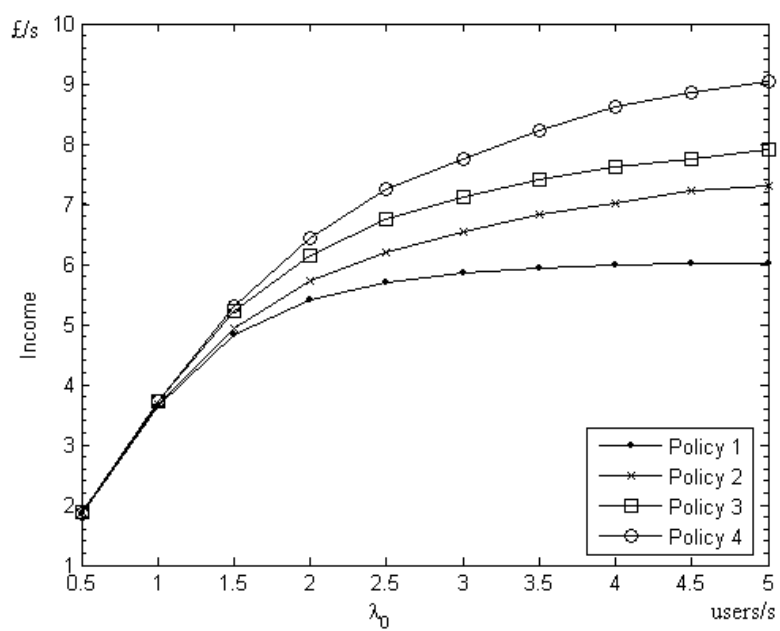

Fig. 3. Total income for the duration of the simulation.

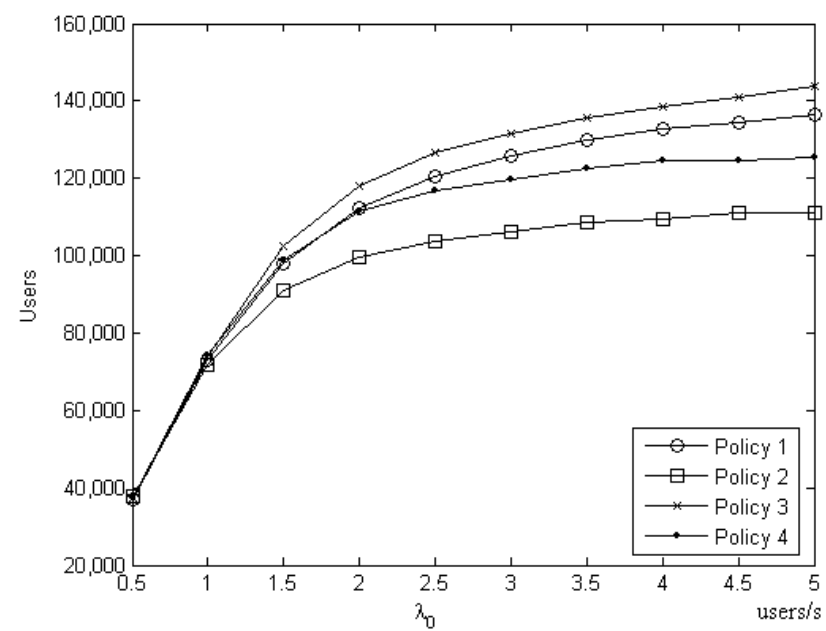

Fig. 4. Total number of accepted users for the duration of the simulation.

potential customers who arrived and didn't find a class that would satisfy them, or, alternatively, the total number of users who actually had a successful call. With Policy 4 the number of accepted users is slightly smaller than with the static policy (Fig. 4). But in Fig. 5 we observe that Policy 4 achieves slightly higher utilisation. The reason is that with Policy 4 more users of higher requirements are being accepted.

Simulations were also conducted for the cases of small networks presented of figures 6 and 7. For the two-link network, with one exception, the dynamic algorithms fare consistently better than the static one, despite the fact that, for

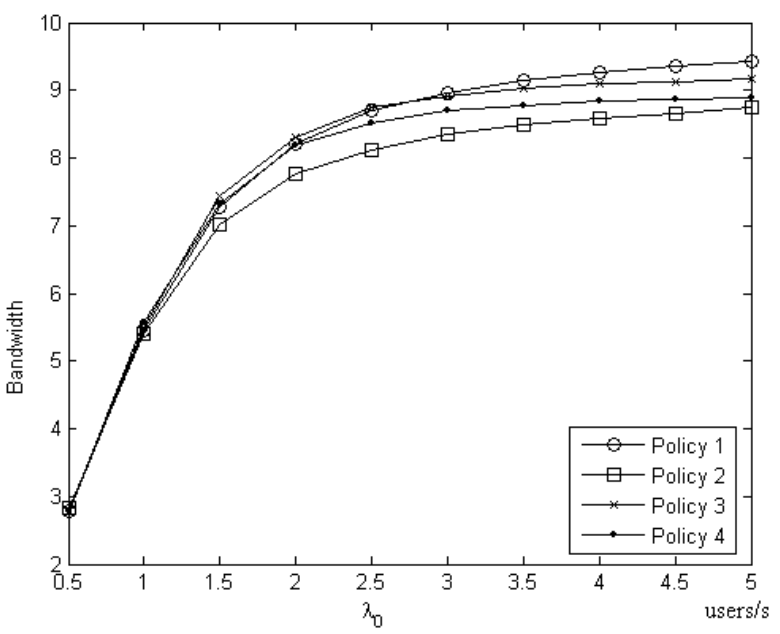

Fig. 5. Average Utilisation.

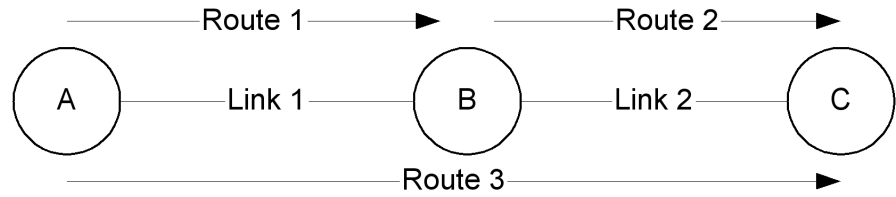

Fig. 6. A simple multi-link case.

the dynamic algorithms, the prices and qualities of each link have been calculated without taking into account the prices and qualities in the other link. This is the main reason why the dynamic quality algorithm (Policy 3 ) has very poor results in case 3 , since in this case the load offered to each link varies greatly, and therefore, as shown in [31], the static solution differs greatly among each link. The problem is that the small link has qualities for its classes which are close to one another and therefore do not leave room for efficient price variations. In fact, whatever advantage is gained locally from varying prices, is severely outweighed by the fact that the high qualities and prices of the small link lead to a much smaller demand on the first link than taken account in the calculation. The main observation is that the dynamic policies, and especially Policy 4 , yield better results compared to Policy 1.

For the same settings, results obtained using the myopic solution, i.e. the solution for which only the next step is taken into account are usually at the same or worse levels than the optimal static ones and therefore, do not offer any practical advantage in the multiple link case. The myopic version of Policy 3 yields the best results among the myopic versions of the four policies, because it combines the optimal static prices 


\begin{tabular}{|c|c|c|c|c|c|c|c|c|c|}
\hline & \multicolumn{5}{|c|}{ Settings } & \multicolumn{4}{|c|}{ Income $(£ / s)$} \\
\hline & \multirow[t]{2}{*}{$W_{1}$} & \multirow[t]{2}{*}{$W_{2}$} & $\lambda_{1}$ & $\lambda_{2}$ & $\lambda_{3}$ & \multicolumn{4}{|c|}{ Policy } \\
\hline & & & \multicolumn{3}{|c|}{ users/s } & 1 & 2 & 3 & 4 \\
\hline Case 1 & 6 & 4 & 11 & 11 & 11 & 5.69 & 5.74 & 5.81 & 6.52 \\
\hline Case 2 & 6 & 4 & 11 & 11 & 21 & 6.08 & 6.27 & 6.17 & 7.14 \\
\hline Case 3 & 10 & 3 & 11 & 11 & 21 & 6.64 & 6.49 & 5.17 & 7.13 \\
\hline Case 4 & 3 & 5 & 11 & 5 & 15 & 4.23 & 4.40 & 4.53 & 4.90 \\
\hline Case 5 & 3 & 5 & 5 & 11 & 15 & 4.66 & 4.85 & 5.11 & 5.50 \\
\hline Case 6 & 3 & 5 & 2 & 2 & 20 & 3.98 & 4.32 & 4.47 & 4.79 \\
\hline
\end{tabular}

TABLE IV

RESUlTS COMPARISON FOR A 2-LINKS NETWORK. BELLMAN EQUATION SOLUTIONS.

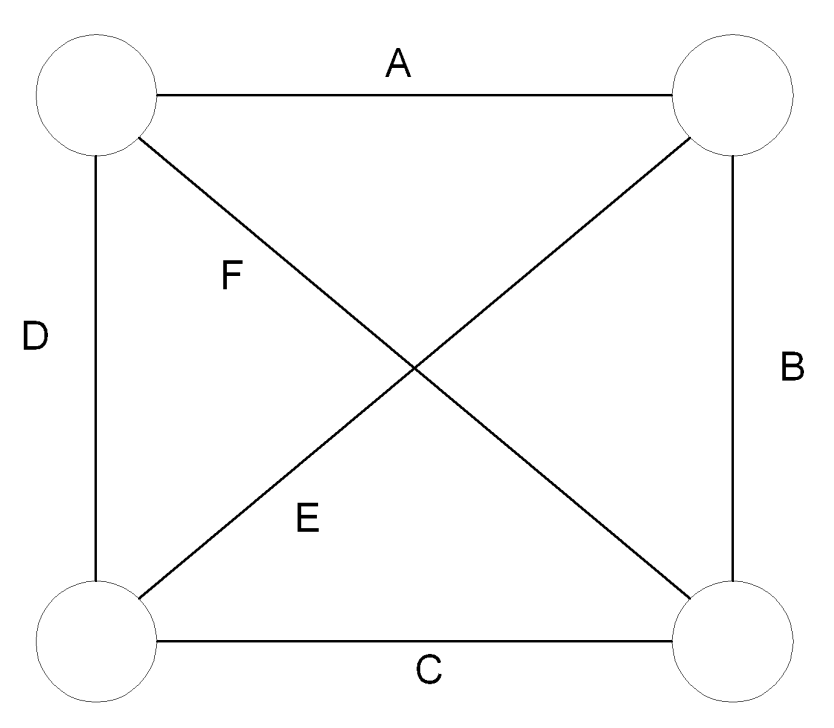

Fig. 7. The square network used for simulations.

with the flexible admission control of the dynamic quality. However, the results for this solution still are inferior to those of the non-myopic dynamic prices and qualities algorithm. An exception to this rule is the dynamic quality/static price myopic policy with results close to and sometimes a little better than the respective optimal/non-myopic policy. The reason for this is the fact that the myopic policies usually consider as optimal higher arrival rates than the optimal ones, since myopic policies don't take into account the fact that accepting many connections now may lead to rejections later. So myopic solutions are characterised by higher expected arrival rates than the optimal. Recall that the most important problem of the decentralised solution is the fact that each link locally does not take into account the conditions in other links in the routes, whose price and quality may "filter" the connections requests, leading to a thinner arrival rate than the one expected. Therefore, when a myopic quality policy is employed, the reduction due to the existence of other links in a route is somewhat offset by the fact that the myopic algorithm leads to higher request arrival rates. In addition to that, the flexibility in quality means that, in case of congestion the quality can be reduced, partially alleviating the effect of not taking into account the problem of potential future blocking. This is an important advantage which does not exist for the

\begin{tabular}{|c|c|c|c|c|c|c|}
\hline & Link A & Link B & Link C & Link D & Link E & Link F \\
\hline Flow 1 & 1 & 1 & 0 & 0 & 0 & 0 \\
Flow 2 & 0 & 1 & 1 & 0 & 0 & 0 \\
Flow 3 & 0 & 0 & 1 & 1 & 0 & 0 \\
Flow 4 & 1 & 0 & 0 & 1 & 0 & 0 \\
Flow 5 & 1 & 0 & 0 & 0 & 1 & 0 \\
Flow 6 & 0 & 1 & 0 & 0 & 0 & 1 \\
Flow 7 & 0 & 0 & 1 & 0 & 1 & 0 \\
Flow 8 & 0 & 0 & 0 & 1 & 0 & 1 \\
Flow 9 & 1 & 0 & 0 & 0 & 0 & 0 \\
Flow 10 & 0 & 1 & 0 & 0 & 0 & 0 \\
Flow 11 & 0 & 0 & 1 & 0 & 0 & 0 \\
Flow 12 & 0 & 0 & 0 & 1 & 0 & 0 \\
Flow 13 & 0 & 0 & 0 & 0 & 1 & 0 \\
Flow 14 & 0 & 0 & 0 & 0 & 0 & 1 \\
\hline
\end{tabular}

TABLE VI

ROUTING MATRIX FOR 4-NODE NETWORK.

case of myopic dynamic pricing, and therefore policies which include myopic dynamic pricing are inferior than the optimal ones.

Similar observations can be made using the four node network of Fig. 7 where the potential routes are given in Table VI. We assume that the potential user mean arrival rate for each of flows 1-4 is $\lambda_{1}$, for flows 5-8 $\lambda_{2}$, for flows 9-12 $\lambda_{3}$ and for flows 13 and $14 \lambda_{4}$, where the indexing of the flows refers to the routing matrix shown in Table VI. The capacity of links A-D is $W_{1}$ and links $\mathrm{E}$ and $\mathrm{F}$ have capacity $W_{2}$. Due to symmetry, the solutions for links A-D will be identical, as well as the solutions for links E-F, making the exhaustive search more tractable. The results for non-myopic and myopic solutions are presented on Tables VII and VIII respectively.

\section{B. Optimal Policy Remarks}

Further results from simulations give us the opportunity to examine the price and quality settings of each of the 4 policies in greater detail. In general, we expect that, under higher demand, the mean offered quality decreases whereas the mean price of the calls of each class increase. However, with Policy 4, there are exceptions to the rule that prices increase and quality decreases with higher demand. To explain this we need to consider the contention among classes for users. In [31] we showed that optimal static policies are such that only one class is satisfying for each user, and this was one of the most interesting results of applying revenue management to telecommunications. The observation under examination here 


\begin{tabular}{|c|c|c|c|c|c|c|c|c|c|}
\hline & \multicolumn{5}{|c|}{ Settings } & \multicolumn{4}{|c|}{ Income $(£ / s)$} \\
\hline & \multirow[t]{2}{*}{$W_{1}$} & \multirow[t]{2}{*}{$W_{2}$} & $\lambda_{1}$ & $\lambda_{2}$ & $\lambda_{3}$ & \multicolumn{4}{|c|}{ Policy } \\
\hline & & & \multicolumn{3}{|c|}{ users/s } & 1 & 2 & 3 & 4 \\
\hline Case 1 & 6 & 4 & 11 & 11 & 11 & 5.69 & 4.63 & 5.81 & 5.37 \\
\hline Case 2 & 6 & 4 & 11 & 11 & 21 & 6.08 & 4.77 & 6.10 & 5.45 \\
\hline Case 3 & 10 & 3 & 11 & 11 & 21 & 6.64 & 5.73 & 5.04 & 6.25 \\
\hline Case 4 & 3 & 5 & 11 & 5 & 15 & 4.23 & 3.41 & 4.53 & 3.86 \\
\hline Case 5 & 3 & 5 & 5 & 11 & 15 & 4.66 & 3.75 & 5.02 & 4.22 \\
\hline Case 6 & 3 & 5 & 2 & 2 & 20 & 3.98 & 3.28 & 4.44 & 3.90 \\
\hline
\end{tabular}

TABLE V

RESUlTS COMPARISON FOR A 2-LINKS NETWORK. MYOPIC SOLUTIONS.

\begin{tabular}{|c|c|c|c|c|c|c|c|c|c|c|}
\hline & \multicolumn{7}{|c|}{ Settings } & \multicolumn{4}{c|}{ Income $(£ / \mathrm{s})$} \\
\cline { 2 - 10 } & $W_{1}$ & $W_{2}$ & $\lambda_{1}$ & $\lambda_{2}$ & $\lambda_{3}$ & $\lambda_{4}$ & \multicolumn{3}{|c|}{ Policy } \\
\cline { 3 - 10 } & & & \multicolumn{3}{|c|}{ users/s } & & 1 & 2 & 3 & 4 \\
\hline Case 1 & 5 & 5 & 5 & 6 & 7 & 3 & 15.97 & 16.39 & 18.22 & 18.82 \\
Case 2 & 8 & 3 & 4 & 5 & 6 & 4 & 18.48 & 18.94 & 19.99 & 21.22 \\
Case 3 & 5 & 3 & 10 & 3 & 2 & 5 & 14.21 & 14.61 & 16.17 & 16.55 \\
Case 4 & 3 & 8 & 4 & 5 & 3 & 5 & 12.15 & 12.39 & 12.90 & 13.94 \\
Case 5 & 10 & 10 & 4 & 2 & 3 & 1 & 18.36 & 18.4 & 17.08 & 19.68 \\
Case 6 & 4 & 4 & 3 & 5 & 3 & 3 & 11.61 & 12.09 & 13.33 & 13.57 \\
\hline
\end{tabular}

TABLE VII

RESULTS COMPARISON FOR 4-NODE NETWORK. BELLMAN EQUATION SOLUTIONS

\begin{tabular}{|c|c|c|c|c|c|c|c|c|c|c|}
\hline & \multicolumn{9}{|c|}{ Settings } & \multicolumn{4}{c|}{ Income $(£ / \mathrm{s})$} \\
\cline { 2 - 10 } & $W_{1}$ & $W_{2}$ & $\lambda_{1}$ & $\lambda_{2}$ & $\lambda_{3}$ & $\lambda_{4}$ & \multicolumn{3}{|c|}{ Policy } \\
\cline { 3 - 10 } & & & \multicolumn{3}{|c|}{ users/s } & 1 & 2 & 3 & 4 \\
\hline Case 1 & 5 & 5 & 5 & 6 & 7 & 3 & 15.97 & 13.08 & 18.30 & 15.34 \\
Case 2 & 8 & 3 & 4 & 5 & 6 & 4 & 18.48 & 16.67 & 20.33 & 18.88 \\
Case 3 & 5 & 3 & 10 & 3 & 2 & 5 & 14.21 & 11.57 & 16.14 & 13.31 \\
Case 4 & 3 & 8 & 4 & 5 & 3 & 5 & 12.15 & 10.66 & 12.99 & 11.92 \\
Case 5 & 10 & 10 & 4 & 2 & 3 & 1 & 18.36 & 17.92 & 17.76 & 19.56 \\
Case 6 & 4 & 4 & 3 & 5 & 3 & 3 & 11.61 & 9.95 & 13.09 & 12.06 \\
\hline
\end{tabular}

TABLE VIII

RESULTS COMPARISON FOR 4-NODE NETWORK. MYOPIC SOLUTIONS

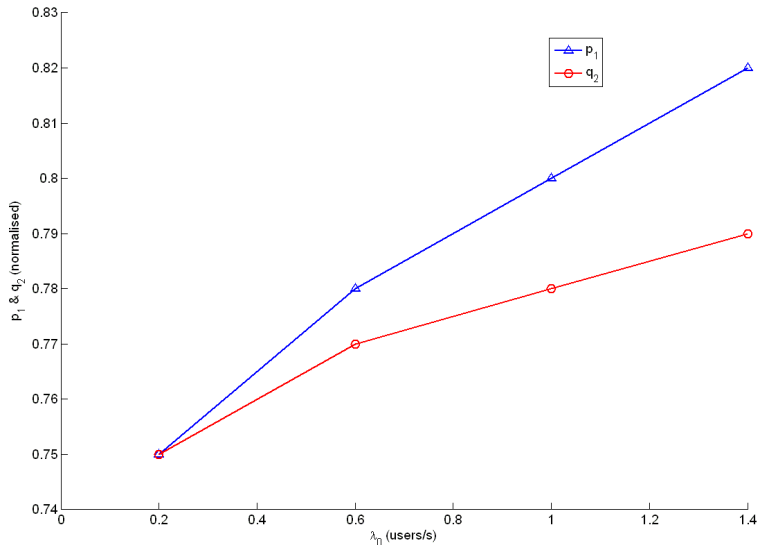

Fig. 8. Normalised price $p_{1}$ of highest class and quality $q_{2}$ of second class as a function of mean total arrival rate of users $\lambda_{0}$. Single link 3 class system, with $B W=5$ and $\mu=0.1$.

is a direct consequence of that. Consider the top two classes of the system. With higher demand, the class of higher quality increases its price and decreases its quality. The increased price $p_{1}$ leaves a segment of potential users unsatisfied by any class. Therefore it may be advisable -in terms of an income maximisation perspective- that the second class $q_{2}$ targets this group of potential users. However, in order to achieve this, its quality has to increase, thus leading to the unexpected result that its average quality $q_{2}$ increases with $\lambda_{0}$ (see Fig. 8).

\section{PRACTICAL CONSIDERATIONS}

The approach proposed here differs from the usual current market practice, where providers post prices which are fairly static, depending largely on demand. Simplicity is a positive thing, but we also must take into account what we are sacrificing for the sake of simplicity. In the past airplane tickets prices were specific for each flight and class. Today, even some car parks and restaurants are priced dynamically, the first depending on demand and utilisation and the second depending on day of the week or even the specific hour the customer sits at the table (not just whether it is lunch or dinner). If such approaches are now beginning to emerge in such industries, which are not inherently technologically advanced, we cannot disregard the potential for their application to telecoms, where technology is more intensely used and therefore, computerbased automation can be applied with more ease, thus making reinforcement learning approaches more feasible, which in turn means that there is no need to obtain information which is not available, such as the demand function. In this paper 
we explore the possibilities and the rewards that a dynamic approach would have to service provisioning.

For the operation of a telecoms network a decision to be made, the decision of price and quality. The alternative to the approach proposed here is an empirical approach, where there is a fuzzy guideline (when demand is high raise prices etc) and the exact decisions are based on that. Our mathematical approach, which is the optimal for the assumptions used. Even if this is not the price which will be used directly, it may be useful to use it as a guide or input other methods of decision making.

\section{FinAl REMARKS}

In this paper we analysed the problem of dynamic resource allocation and pricing, with the aim of income maximisation. An optimal dynamic control of pricing and quality has been proposed. This proposal is within the spirit of revenue management, where decisions regarding pricing and quality are influenced by the available resources and demand. The conclusion that can be drawn from the work reported in this paper, is that the application of revenue management principles in resource allocation and pricing in a telecommunications network environment may lead to an improvement of a provider's results. This opens the road for further research in this direction and in particular addressing some of the outstanding research issues here highlighted.

To make our approach more applicable, the integration of our pricing and resource allocation approach with current market practices and conditions has to be considered. Such issues include the existence of competition, which may be studied by introducing and studying a game-theory approach and the bundling of services. The latter could be tackled by appropriately modifying the reward function of the provider (accepting a user might yield more income than just the fees for internet services) and the demand function of the users.

In order to further enhance the scalability of the proposed approach, in Section VI.C we suggest to further study the conditions under which the proposed procedure is optimal and developing automated methods for selecting the appropriate policy type for each link. Finally, in Section V.C we propose to further investigate the criteria to aggregate state of the network an its associated loss in revenue minimisation, in the case that the dynamics of the network and real time constraints to the solutions becomes an issue.

\section{Acknowledgements}

The authors would like to thank the referees for their most helpful and constructive comments.

\section{REFERENCES}

[1] H. Che, S. Zheng, X. Hong, A model analysis of pricing and link bandwidth allocation in a multiple class-of-service network, Proceedings of IEEE International Conference on Computer Communications and Networks, 2000, pp. 510-516.

[2] H. Che, S. Zheng, X. Hong, Integrated model for performance analysis of multiple class-of-service Internet, IEE Proceedings in Communications, Vol. 149, No. 3, 2002, pp. 139-146. agent.ps.gz.

[3] R. G. Cross, Revenue Management, Hard Core Tactics for Market Domination, Broadway Books, New York, 1998.
[4] R. Edell and R. Varaiya, Demand for quality-differentiated network services, in Proc. 36th IEEE Conf. on Decision and Control, San Diego, CA, Dec. 1997, pp. 1443-1448.

[5] E. W. Fulp, D. S. Reeves, Bandwidth provisioning and pricing for networks with multiple classes of service, Computer Networks, Vol. 46, No. 1, 2004, pp. 41-52.

[6] Y. Hayel, B. Tuffin, A mathematical analysis of the cumulus pricing scheme, Computer Networks, Vol. 47, No. 6, 2005, pp. 907-921.

[7] S. Humair, Yield Management for Telecommunication Networks: Defining a New Landscape, PhD Thesis, MIT, 2001.

[8] F. P. Kelly, Notes on Effective Bandwidths, Stochastic Networks: Theory and Applications, 1996, pp. 141-168.

[9] N. J. Keon, G. Anandalingam, Optimal pricing for multiple services in telecommunications networks offering quality-of-service guarantees, IEEE/ACM Transactions on Networking, Vol. 11, No. 1, 2003, pp. 6680.

[10] J. O. Kephart, J. E. Hanson and A. R. Greenwald, Dynamic pricing by software agents, Computer Networks, Vol. 32, No. 6, 2000, pp. 731-752.

[11] T. Li, Y. Iraqi, R. Boutaba, Pricing and admission control for QoSenabled Internet, Computer Networks, Vol. 46, No. 1, 2004, pp. 87-110.

[12] J. K. MacKie-Mason, H. R. Varian, Pricing congestible network resources, IEEE Journal on Selected Areas in Communications, Vol. 13, No. 7, 1995, pp. 1141-1149.

[13] D. Mayer, J. A. Barria, Bandwidth Allocation for a Revenue-Aware Network Utility Maximisation, IEEE Communications Letters, Vol. 11, No. 7, 2007, pp.634-636.

[14] A. Odlyzko, Internet pricing and the history of communications, Computer Networks, Vol. 36, No. 5-6, 2001, pp. 493-517.

[15] I. Ch. Paschalidis, J. N. Tsitsiklis, Congestion-dependent pricing of network services, IEEE/ACM Trans. on Networking, Vol. 8, No. 2, 2000, pp. 171-184.

[16] I. Ch. Paschalidis, L. Yong, Pricing in multiservice loss networks: static pricing, asymptotic optimality and demand substitution effects, IEEE/ACM Transactions on Networking, Vol. 10, No. 3, 2002, pp. 425438

[17] I. Ch. Paschalidis, L. Yong, Distributed resource allocation in multiservice communication networks using pricing, in Proceedings of the $41 \mathrm{st}$ IEEE Conference on Decision and Control, Vol. 2, 2002, pp. 2023-2028.

[18] A. Patel, K. Prouskas, J. Barria, J. Pitt, A Computational Economy for IN Load Control using Multi-agent System, Journal of Network and Systems Management, Vol. 8, No. 3, 2000, pp. 397-417.

[19] J. M. Peha, Dynamic pricing and congestion control for best-effort ATM services, Computer Networks, Vol. 32, No. 3, 2000, pp. 333-345.

[20] K. Prouskas, A. Patel, J. Pitt, J. Barria, A Multi-Agent System for Intelligent Network Load Control Using a Market-Based Approach, Fourth International Conference on Multi-Agent Systems, 2000, pp. 231238.

[21] P. Reichl, D. Hausheer and B. Stiller, The Cumulus Pricing model as an adaptive framework for feasible, efficient, and user-friendly tariffing of Internet services, Computer Networks, Vol. 43, No. 1, 2003, pp. 3-24.

[22] D. Ros and B. Tuffin, A mathematical model of the Paris Metro Pricing scheme for charging packet networks, Computer Networks, Vol. 46, No. 1, 2004, pp. 73-85.

[23] B. Rupp, R. Edell, H. Chand, P. Varaiya, INDEX: A Platform for Determining how People Value the Quality of their Internet Access, Proceedings of the 6th IEEE/IFIP IWQoS, May 1998, pp. 85-90.

[24] J. Sairamesh, J. O. Kephart, Price Dynamics of Vertically differentiated information markets, Proceedings of the first international conference on Information and computation economies, 1998, pp. 28-36.

[25] R. M. Salles and J. A. Barria, Fair and efficient dynamic bandwidth allocation for multi-application networks, Computer Networks, Vol. 49, No. 6, 2005, pp. 856-877.

[26] N. Semret, R. R.-F. Liao, A. T. Campbell, A. A. Lazar, Pricing, provisioning and peering: dynamic markets for differentiated Internet services and implications for network interconnections, IEEE Journal on Selected Areas in Communications, Vol. 18, No. 12, 2000, pp. 24992513.

[27] N. Semret, R. R.-F. Liao, A. T. Campbell, A. A. Lazar, Peering and provisioning of differentiated internet services, in Proceedings of INFOCOM 2000, Vol. 2, 2000, pp. 414-420.

[28] R. S. Sutton, A. G. Barto, Reinforcement Learning:An Introduction, MIT Press, Cambridge, MA, 1998.

[29] X. Wang, H. Schulzrinne, An Integrated resource negotiation, Pricing, and QoS Adaptation Framework for multimedia applications, IEEE Journal on Selected Areas in Communications, Vol. 18, No. 12, 2000, pp. 2514-2529. 
[30] J. Youngmi, G. Kesidis, Dynamics of usage-priced communication networks: the case of a single bottleneck resource, IEEE/ACM Transactions on Networking, Vol. 13, No. 5, 2005, pp. 1041-1053.

[31] G. Zachariadis, J. A. Barria, Demand management for telecommunications services, Computer Networks, Vol. 51, No 12, 2007, pp. $3507-$ 3524.

[32] G. Zachariadis, J. Barria, Dynamic revenue management for flows in packet networks, in Proceedings of IEEE ICC 2007, pp. 2076-2081.

[33] Usaha, W., Barria, J.A. (2007). Reinforcement learning for resource allocation in LEO satellite networks. IEEE Transactions on Systems, Man, and Cybernetics, B37(3), 515-527.

[34] Usaha, W., Barria J.A. (2007). QoS Routing in MANET with Imprecise Information Using Actor-Critic Reinforcement Learning, IEEE WCNC 2007, 3382-3387.

[35] Usaha, W. and Barria, J., "A Markov Decision Theory Framework for Resource Allocation in LEO Satellite Constellations", IEE Proc.Commun., Vol 149, No 5, (2002), pp. 270-276. 